\title{
TROCHANTERIC GROWTH DISTURBANCE AFTER UPPER FEMORAL OSTEOTOMY FOR CONGENITAL DISLOCATION OF THE HIP
}

\author{
C. B. SCHOFIELD, J.G. SMIBERT
}

From Queen Mary's Hospital for Children, Carshalton

\begin{abstract}
We reviewed 14 patients (16 hips) treated by open reduction and upper femoral derotation osteotomy for congenital dislocation of the hip. Nine patients with 11 treated hips had growth deformities of the proximal femur; in all of them the top screw of the fixation plate lay within the cartilaginous precursor of the greater trochanter. In the five control hips the top screw was more distal.

In the nine patients (mean follow-up 10.8 years) there was an increase of $14^{\circ}$ in the neck-shaft angle $(p=0.01)$ and of $18^{\circ}$ in the angle between the capital femoral physis and the shaft $(p=0.01)$ compared to the control group. This indicates that growth disturbance of the greater trochanteric apophysis as a result of plate fixation leads to long-term deformity.
\end{abstract}

Several abnormalities of the femoral neck and greater trochanter have been described after treatment for congenital dislocation of the hip (CDH). These include deformities of the greater trochanter, anteversion of the femoral neck and valgus and varus deviations. Compere, Garrison and Fahey (1940) showed in goats that surgical trauma to the growth plate of the greater trochanter resulted in growth arrest with deformity and shortening of the trochanter, and sometimes associated coxa valga of the femoral neck. Langenskiöld and Salenius (1967) reported two cases of progressive coxa valga after damage to the trochanteric physis, and used this evidence to suggest trochanteric epiphysiodesis to treat coxa vara.

Laurent (1959) reported the results of removing a bone and cartilage graft from the greater trochanter for use in acetabular augmentation for $\mathrm{CDH}$ : in some cases coxa valga was seen to develop. Jones (1977) reviewed six cases of subcapital coxa valga secondary to varus osteotomy but was unable to relate this to physeal damage of the greater trochanter. He suggested that poor acetabular cover was a possible factor.

C. B. Schofield, FRCS, Lecturer in Orthopaedic Surgery.

Department of Orthopaedics, Charing Cross Hospital (Fulham), Fulham Palace Road, London W6 8RF, England.

J. G. Smibert, FRCS, Senior Orthopaedic Registrar

King's College Hospital, Denmark Hill, London SE5 9RS, England.

Correspondence should be sent to $\mathrm{Mr}$ C. B. Schofield.

(C) 1989 British Editorial Society of Bone and Joint Surgery

$0301-620 X / 90 / 1198 \$ 2.00$

J Bone Joint Surg [Br] 1990; 72-B: 32-6.

\section{PATIENTS AND METHODS}

From 1967 to 1987, 27 patients presented with late dislocation of the hip at Queen Mary's Hospital for Children, Carshalton. Of these, 16 have been followed up radiologically for a mean of 11.8 years. All were originally treated by open reduction followed after an interval by upper femoral varus derotation osteotomy. Two types of internal fixation were used: an angled fourhole plate, or a Coventry screw plate.

Postoperative radiographs of nine of these patients showed that the top screw in the plate was lying within the cartilaginous precursor of the greater trochanter. To assess the effect of this we identified two groups of patients and compared their radiographs.

Group A. Nine patients with 11 affected hips showed growth problems secondary to disturbance of the growth plate of the greater trochanter. Their mean age at operation was 24 months, and mean follow-up was 10.8 years; there were seven girls and two boys.

Group B. Five patients without screw or plate in the trochanter were used as controls: they had no adverse growth effects. Their mean age at operation was 20.6 months, mean follow-up was 13.2 years; and all were girls. Four of the five osteotomies had been fixed by the Coventry screws and one by an angled plate which was not lying across the physis of the greater trochanter.

Two patients (three hips) were excluded because of evidence of avascular necrosis, acetabular dysplasia or technical problems associated with reduction. 


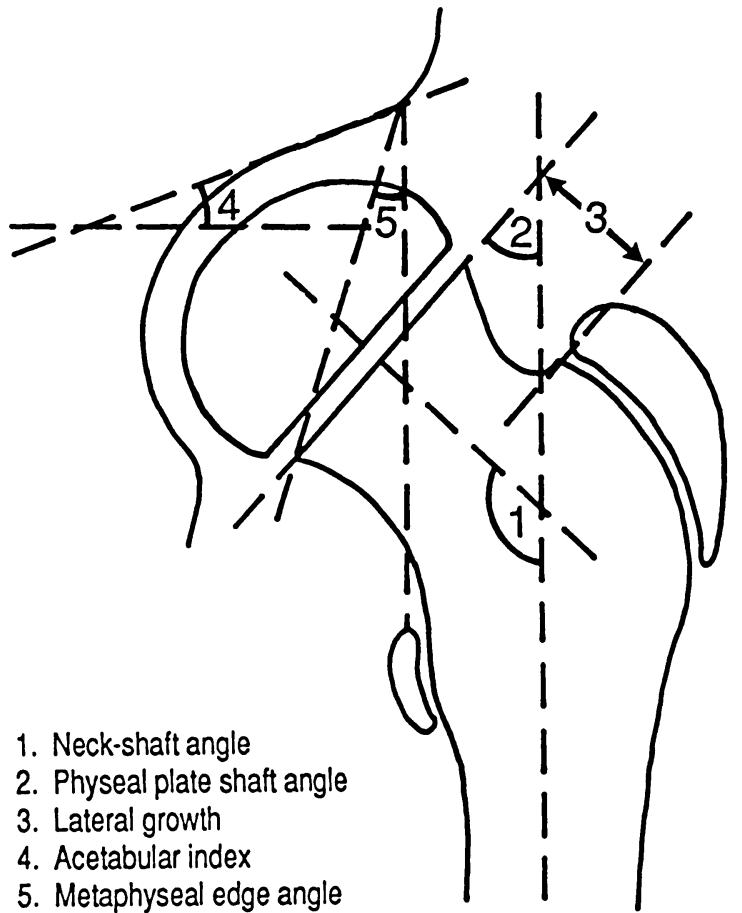

Fig. 1

Radiological measurements.

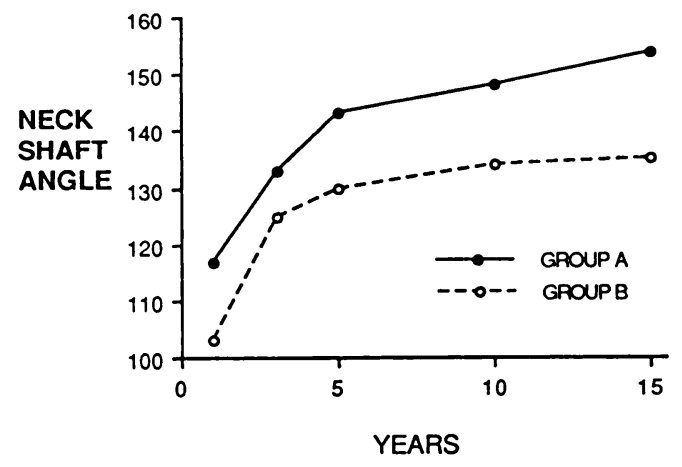

Fig. 2

Changes in neck-shaft angle related to years since operation. Group A had greater trochanteric growth disturbance, group B were controls.

At radiological follow-up of both groups, the following measurements were made on standard plain anteroposterior radiographs (Fig. 1) at one, three, five, 10 and 15 years after operation:

1) Neck-shaft angle;

2) Angle between capital physeal plate and the shaft (Jones 1977);

3) Growth of the lateral aspect of the femoral neck;

4) Metaphyseal angle (Dyson, Lynskey and Catterall 1987);

5) Acetabular index.
The lateral growth of the femoral neck was estimated directly by measuring the distance on a plain anteroposterior radiograph between the most superolateral point of the capital femoral metaphysis and the point where the greater trochanter meets the lateral aspect of the femoral neck.

Statistical analysis used an unpaired Student's $t$-test and the Wilcox's sum of rank test.

\section{RESULTS}

These are summarised in Table I. Patient records revealed no differences in operative technique except for the method of internal fixation of the osteotomy, and the varus angulation produced at operation was similar in both groups.

Neck-shaft angle. There was an increase in the neckshaft angles in group A even at one year (Fig. 2). By 10 years the difference was significant $(p=0.01)$. The neckshaft angle could not be measured immediately after operation because poor radiographs had been taken through plaster in non-standard positions. Group A patients show an increasing neck-shaft angle up to skeletal maturity, while patients in group B had a normal growth pattern with a final angle of $135^{\circ}$.

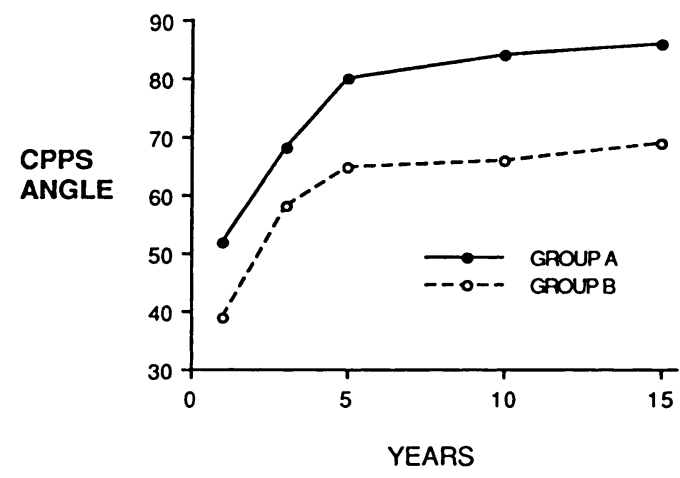

Fig. 3

Changes in angle between the capital physeal plate and the femoral shaft.

Angle between capital physis and shaft. Group A patients again showed a significant increase at 10 years $(p=0.01)$ (Fig. 3). The angle at 10 years was $18^{\circ}$ higher in group $\mathrm{A}$, indicating a more horizontal growth plate.

Growth of the lateral femoral neck. Lateral growth of the femoral neck, was measured directly from radiographs (Fig. 1), and the earliest reliable results were obtained three years after operation and used as a baseline for further growth. Growth was found to be significantly lower in group A than in group $B(p=0.10)$ (Fig. 4).

Metaphyseal edge angle and acetabular index. Our 
measurements showed no difference between the two groups, which suggests that growth disturbance of the greater trochanter had no effect on acetabular development (Figs 5 and 6).

At 15 years after operation few hips were available: only four from group A and three from group B, but this data has been included since the results correlate well. The radiological measurements and the radiographs at each time interval confirm the typical appearances seen in group A (Fig. 7). Typically, coxa valga is associated with a horizontal capital physeal plate. The femoral neck is long and slender and the shape of the greater trochanter is abnormal, giving the appearance of being tethered to the lateral aspect of the femoral neck.

Three patients (three hips) in group A required a second operation at four years, four years and five years respectively after the primary procedure; two had further upper femoral varus osteotomies and one had a Salter innominate osteotomy to control the degree of coxa valga. At latest review all three patients had a normal neckshaft angle with good containment of the femoral head. At the time of the secondary procedures, all three patients had normal acetabular indices. The radiological measurements of these three hips after secondary operations were not included in the main series.

\section{DISCUSSION}

At birth the proximal femur is covered by a continuous cartilaginous plate which is destined to form both the capital epiphysis and the greater trochanteric apophysis. Damage and growth disturbance in either of these regions will lead to a related anatomical abnormality in the other. This provides a potential problem until the age of 16 to 19 years, when the two physes finally close. Our study supports previous research into the effects of damage to the physis of the greater trochanter.

Compere et al (1940) showed that growth arrest of the greater trochanteric physis in goats resulted in an abnormal shape of the greater trochanter and coxa valga. Laurent (1959) confirmed these results in rabbits and also observed that a long slender femoral neck developed. Some of our radiological results could result from femoral neck anteversion, but Ewald and Hirohashi (1973) failed to show any change in anteversion after distal transfer of the greater trochanter in dogs.

In our study the re-operation rate was $18.8 \%$. Williamson and Benson (1988) reported a re-operation rate of $19 \%$ in 502 cases of congenital dislocation of the hip treated by upper femoral varus derotation osteotomy; three-quarters of their patients had a coxa valga deformity and also a significant incidence of acetabular growth abnormalities. They concluded that an acetabular index greater than $27 \%$ led to a poor result. Both groups in our study had normal acetabular indices.

Subcapital coxa valga after varus osteotomy for congenital dislocation of the hip was reported by Jones
(1977) in six patients with a mean follow-up of nine years. Jones concluded that damage to the greater trochanteric physis could not be identified as an aetiological factor, but did find an association between poor acetabular cover and coxa valga. We excluded patients with evidence of acetabular growth abnormalities and found that radiological indices of acetabular development were not significantly different between our two groups.

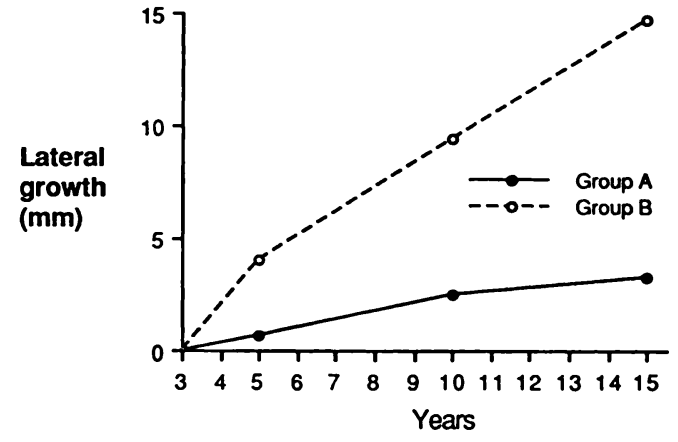

Fig. 4

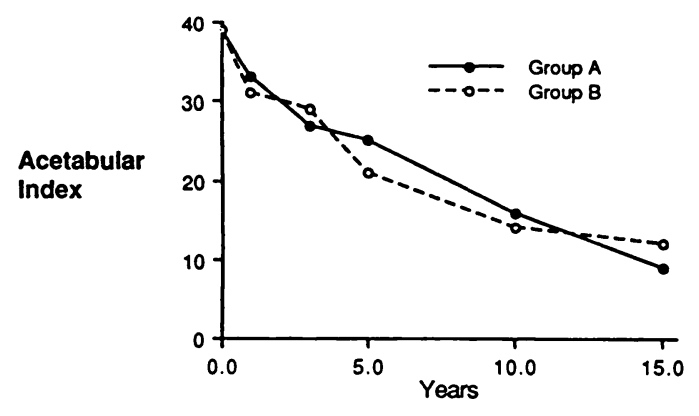

Fig. 5

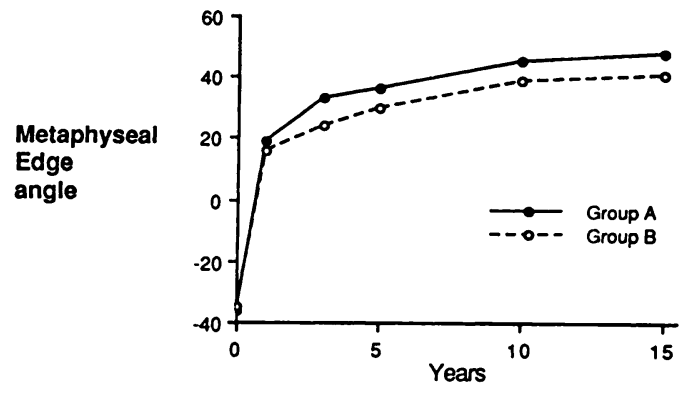

Fig. 6

Changes in lateral growth, acetabular index and metaphyseal edge angle related to years since operation.

The growth abnormalities we found must have been caused by plate fixation across the greater trochanteric physis since this is the only significant difference between the two groups. We have further examined the complex relationship between the greater trochanteric physis and 
Table I. Results of radiological measurements in degrees

\begin{tabular}{lllllllll}
\hline & \multicolumn{7}{c}{ Years after operation } \\
\cline { 4 - 9 } & Group & $\mathbf{0}$ & $\mathbf{1}$ & $\mathbf{3}$ & $\mathbf{5}$ & 10 & 15 \\
\hline Physeal plate shaft angle & A & & 52 & 68 & 80 & 84 & 86 \\
& B & & 39 & 58 & 65 & 66 & 69 \\
& p value & & NS & NS & 0.05 & 0.01 & $*$ \\
Neck-shaft angle & A & & 117 & 133 & 143 & 148 & 154 \\
& B & & 103 & 125 & 130 & 134 & 135 \\
& p value & & NS & NS & 0.05 & 0.01 & $*$ \\
Acetabular index & A & 39 & 33 & 27 & 25 & 16 & 9 \\
& B & 39 & 31 & 29 & 21 & 14 & 12 \\
& p value & NS & NS & NS & NS & NS & $*$ \\
Metaphyseal shaft angle & A & -36 & 19 & 33 & 36 & 45 & 48 \\
& B & -35 & 16 & 24 & 30 & 39 & 41 \\
& p value & NS & NS & NS & NS & NS & $*$ \\
\hline
\end{tabular}

* small numbers of patients at 15 years

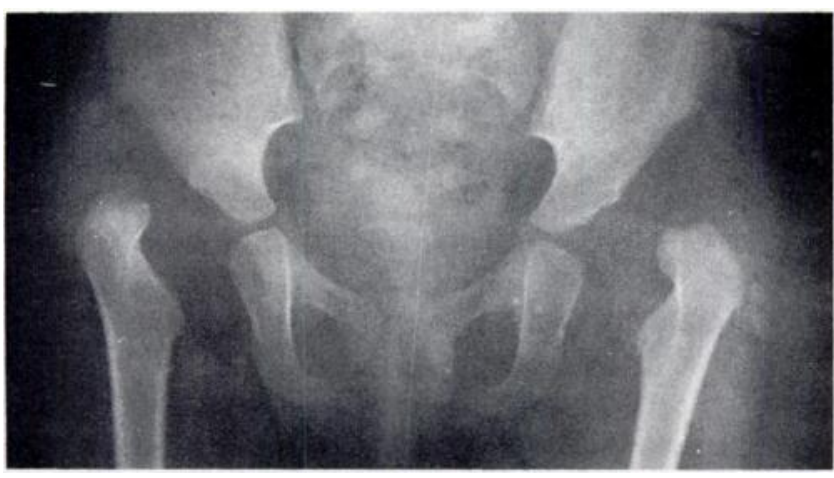

Fig. 7a

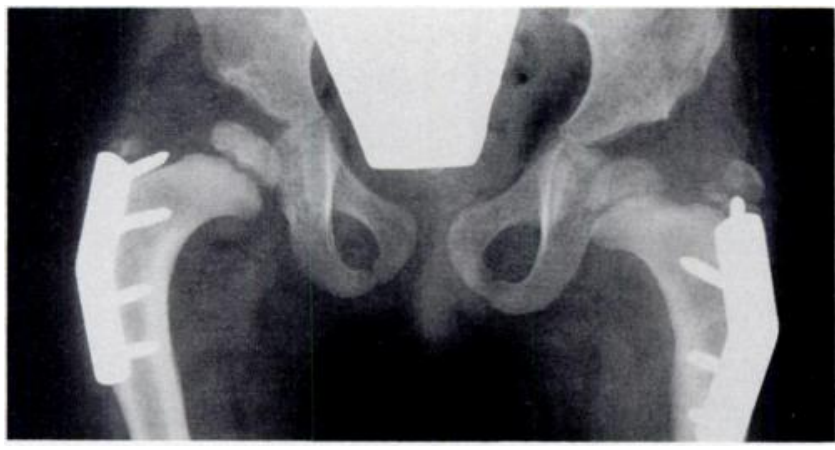

Fig. 7c

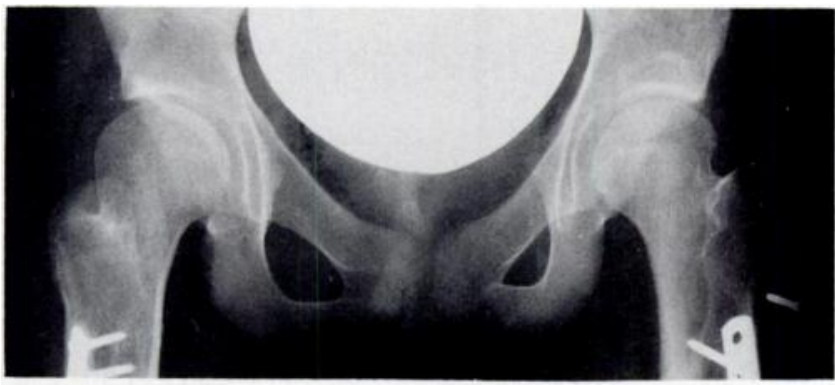

Fig. 7e

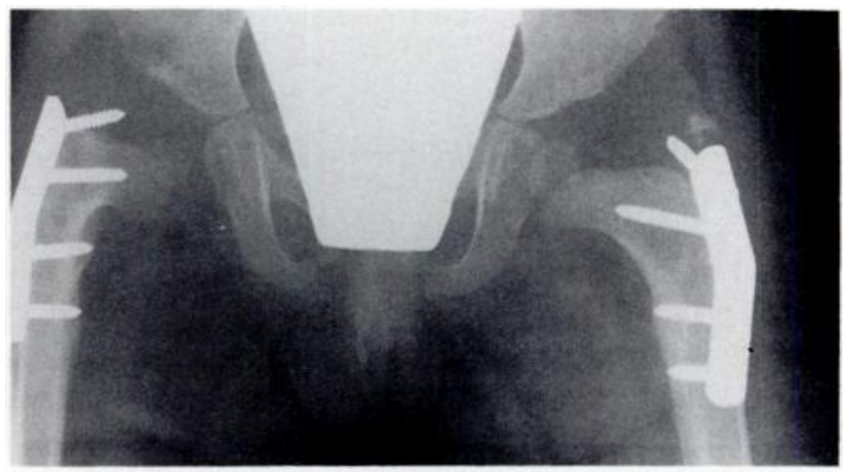

Fig. 7b

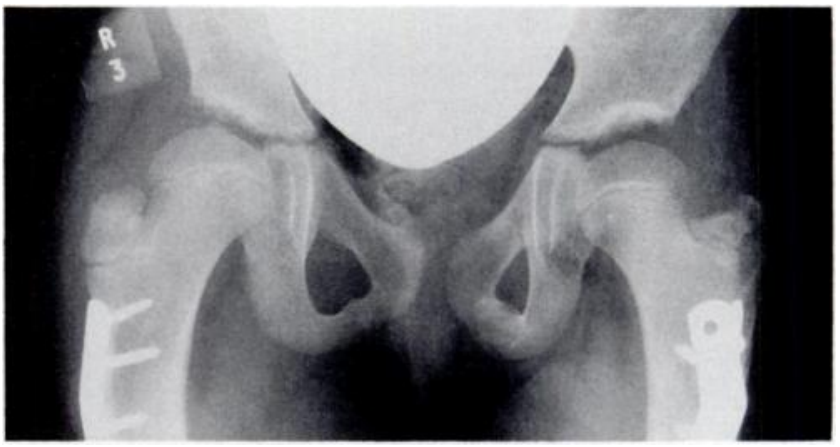

Fig. 7d

Radiographs of a patient developing bilateral coxa valga after the proximal screw crossed the greater trochanteric physis. Films taken before operation (a) and at three (b), five (c), 10 (d) and 15 years follow-up (e) 
the capital femoral physis and our results suggest that most of the growth disturbance occurred within the first year after fixation. Once lateral tethering has taken place the more horizontal capital physis produces an increased neck- shaft angle and coxa valga. The long slender femoral neck observed in group A patients may have been produced by an alteration in the load distribution on the capital physis, leading to stimulation of longitudinal growth in the femoral neck.

Our study has confirmed in patients the results of damage to the greater trochanteric physis seen in previous animal experiments. Whereas damage to the capital femoral epiphysis generally causes a short varus femoral neck, damage to the trochanteric physis leads to a long valgus femoral neck without significant disturbance of acetabular development.

We wish to extend our thanks to Mr G. F. Walker and Mr P. CheongLeen for allowing us to report on their cases, $\mathrm{Mr} \mathrm{A}$. Catterall for reading the manuscript and Miss M. Sellar for typing the manuscript.

No benefits in any form have been received or will be received from a commercial party related directly or indirectly to the subject of this article.

\section{REFERENCES}

Compere EL, Garrison M, Fahey JJ. Deformities of the femur resulting from arrestment of growth of the capital and greater trochanteric epiphyses. J Bone Joint Surg 1940; 22:909-15.

Dyson PHP, Lynskey TG, Catterall A. Congenital hip dysplasia: problems in the diagnosis and management of the abnormal hip in the first year. J Bone Joint Surg [ Br] 1987; 69-B:155.

Ewald FC, Hirohashi K. Effect of distal transfer of the greater trochanter in growing animals. J Bone Joint Surg [ Am] 1973; 55-A:1064-7.
Jones DA. Sub-capital coxa valga after varus osteotomy for congenital dislocation of the hip: a report of six cases with a minimum followup of nine years. J Bone Joint Surg [Br] 1977; 59-B:152-8.

Langenskiöld A, Salenius P. Epiphyseodesis of the greater trochanter. Acta Orthop Scand 1967; 38:199-219.

Laurent LE. Growth disturbances of the proximal end of the femur in the light of animal experiments. Acta Orthop Scand 1959; $28: 255-61$.

Williamson DM, Benson MKD. Late femoral osteotomy in congenital dislocation of the hip. J Bone Joint Surg [ Br] 1988; 70-B:614-8. 